\title{
Charge screening in classical scalar electrodynamics
}

\author{
M. Bawin and J. Cugnon \\ Institut de Physique B5, Université de Liège, Sart Tilman, B-4000 Lì̀ge 1, Belgium
}

(Received 26 October 1987)

\begin{abstract}
We consider the problem of a fixed source of charge $Z$ in a classical electromagnetic interaction with a scalar field of mass $m$. We find that partially screened solutions with energy less than the energy associated with pure Coulomb configurations (with zero scalar field) exist for $Z>Z_{0}$, where $Z_{0}$ is the value at which the Klein-Gordon equation for a particle of mass $m$ in the external Coulomb field of the source has zero-frequency solutions. Our model thus allows for the exact construction of a classical "charged vacuum."
\end{abstract}

We recently studied the (nonlinear) problem of a fixed source of charge $Z$ in interaction with a charged scalar field of mass $m$ within the framework of classical electrodynamics. $^{1}$ We found, as expected on general grounds, ${ }^{2}$ that there exists a critical charge $Z=Z_{\mathrm{cr}}$ such that, for $Z>Z_{\text {cr }}$, the Coulomb solution (i.e., the bare source configuration with zero scalar field) no longer is the solution with minimum energy. Indeed, we explicitly constructed for $Z>Z_{\text {cr }}$ partially screened solutions (with nonzero scalar field) with energy less than the energy $E_{C}$ associated with a pure Coulomb solution. The critical value $Z=Z_{\mathrm{cr}}$ in this problem is determined by that value of $Z$ at which the linear Klein-Gordon equation for a particle of mass $m$ in an external Coulomb field of charge $Z$ yields an eigenvalue $\omega=-m$.

We were motivated to further study this problem by a recent paper on the instability of large- $Z$ nuclei with respect to electron-positron pair creation, ${ }^{3}$ a problem of great current interest. ${ }^{4}$ The instability of such superheavy nuclei is expected to occur at $Z \simeq 170$, i.e., the value at which the single-particle Dirac equation has eigenstates with $\omega=-m_{e}$ eigenvalues ( $m_{e}$ is the electron mass). According to the authors of Ref. 3, electron-positron pair creation could occur at $Z \simeq 150$, which roughly corresponds to $\omega=0$ eigenvalues of the Dirac equation. These authors further argue that, contrary to current belief, 5 the ground state of the system is not correctly described by a "charged vacuum." In view of the many approximations used in the quantum-field-theoretic study in Ref. 3, we have deemed of interest to study in more detail whether a similar result could be obtained within a simple exactly soluble model such as classical electrodynamics with an external source. Our main result is the following. The value of the external charge for which partially screened solutions, with charge $Q<Z$, start having a lower energy than the pure Coulomb solution is indeed given by $Z=Z_{0}<Z_{\text {cr }}$, where the value $Z_{0}$ is the $Z$ value at which the Klein-Gordon equation for a particle of mass $m$ in an external Coulomb field has zero eigenvalues. However, as discussed in Ref. 2, this does not mean that the pure Coulomb solutions are unstable for $Z_{0}<Z<Z_{\text {cr }}$. Only for $Z>Z_{\text {cr }}$ does one expect them to become unstable, as the Klein-Gordon equation then has complex eigenvalues. ${ }^{2}$
In order to see this in detail, we start with the equations of motion for a charged scalar field $\phi$ in interaction with the electromagnetic field $A^{\mu}$ in in the presence of an external source $j_{\mu}^{\text {ext }}$ :

$$
\begin{aligned}
& \left(\partial_{\mu}-i e A_{\mu}\right)^{2} \phi+m^{2} \phi=0, \\
& \square A_{\mu}-\partial_{\mu} \partial^{v} A_{v}+i e\left(\phi^{*} \overleftrightarrow{\partial}_{\mu} \phi-2 i e A_{\mu} \phi^{*} \phi\right)=j_{\mu}^{\text {ext }},
\end{aligned}
$$

where

$$
\alpha \equiv \frac{e^{2}}{4 \pi}=\frac{1}{137} .
$$

Taking, as in Ref. 6,

$$
j_{\mu}^{\mathrm{ext}}=\frac{e Z \delta\left(r-r_{0}\right)}{r^{2}} \delta_{\mu 0},
$$

i.e., considering a static charge distribution with density

$$
\rho(r) \equiv \frac{e Z \delta\left(r-r_{0}\right)}{r^{2}}
$$

and working in the radiation gauge $\boldsymbol{\nabla} \cdot \mathbf{A}=0$, one finds, from (1), (2), and (4),

$$
\begin{aligned}
& \frac{d^{2} g}{d r^{2}}+\frac{f^{2}-m^{2} r^{2}}{r^{2}} g=0, \\
& \frac{d^{2} f}{d r^{2}}-\frac{g^{2} f}{r^{2}}=-\frac{\alpha Z}{r_{0}} \delta\left(r-r_{0}\right),
\end{aligned}
$$

provided we look for solutions of the form ${ }^{1}$

$$
\begin{aligned}
& \phi(r)=e^{i \omega t} \frac{g}{\sqrt{2} e r}, \\
& e A_{0}=\frac{f}{r}-\omega, \\
& \mathbf{A}=0 .
\end{aligned}
$$

The total energy $E$ associated with a given solution is given by

$$
E=4 \pi \int_{0}^{\infty} T_{00} r^{2} d r,
$$

where 


$$
\begin{aligned}
T_{00}= & \frac{f^{2} g^{2}}{2 e^{2} r^{4}}+\frac{1}{2 e^{2}}\left[\left(\frac{g}{r}\right]^{\prime}\right]^{2} \\
& +\frac{m^{2}}{2 e^{2}} \frac{g^{2}}{r^{2}}+\frac{1}{2 e^{2}}\left[\left(\frac{f}{r}\right]^{\prime}\right]^{2} .
\end{aligned}
$$

From (5) and (6), we find, in the linear approximation ${ }^{1}$ $(g \simeq \widetilde{g})$,

$$
\left[\frac{d^{2}}{d r^{2}}+\left(e A_{0}-\omega\right)^{2}-m^{2}\right) \widetilde{g}=0
$$

with

$$
\begin{aligned}
& A_{0}=\frac{e Z}{4 \pi r} \quad\left(r>r_{0}\right), \\
& A_{0}=\frac{e Z}{4 \pi r_{0}} \quad\left(r<r_{0}\right) .
\end{aligned}
$$

In contrast with Ref. 1, we now look for solutions to (5) and (6) with an arbitrary frequency $\omega$, i.e., with boundary conditions at infinity:

$$
\begin{aligned}
& f(r) \underset{r \rightarrow \infty}{\simeq} \omega r+\alpha Q, \\
& g(r) \underset{r \rightarrow \infty}{\simeq} C \exp \left[-\left(m^{2}-\omega^{2}\right)^{1 / 2} r\right] .
\end{aligned}
$$

The boundary conditions at the origin remain unchanged

$$
\begin{aligned}
& f(r) \underset{r \rightarrow 0}{\simeq} f_{1} r, \\
& g(r) \underset{r \rightarrow 0}{\simeq} g_{1} r .
\end{aligned}
$$

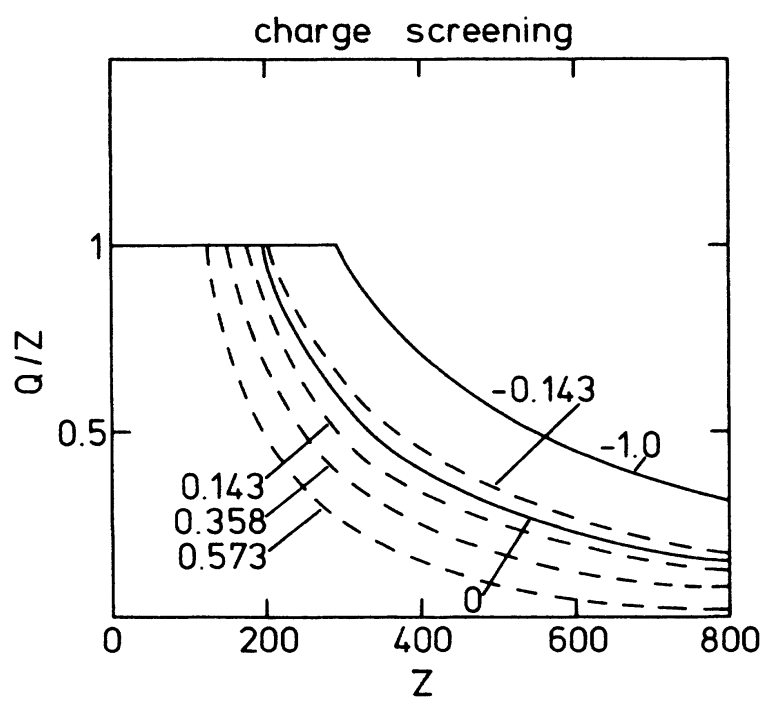

FIG. 1. Values of the total charge $Q$ as a function of the external charge $Z$. The indicated numbers give the different values of $\omega / \mathrm{m}$. In particular, the curve labeled -1.0 corresponds to $\omega / m=-1$ and starts to depart from unity at $Z=Z_{\mathrm{cr}}=290$, while the curve labeled 0 corresponds to $\omega=0$ and $Z=Z_{0}=190$. All the curves shown have been calculated for the parameter $x=m r_{0}=0.567$.

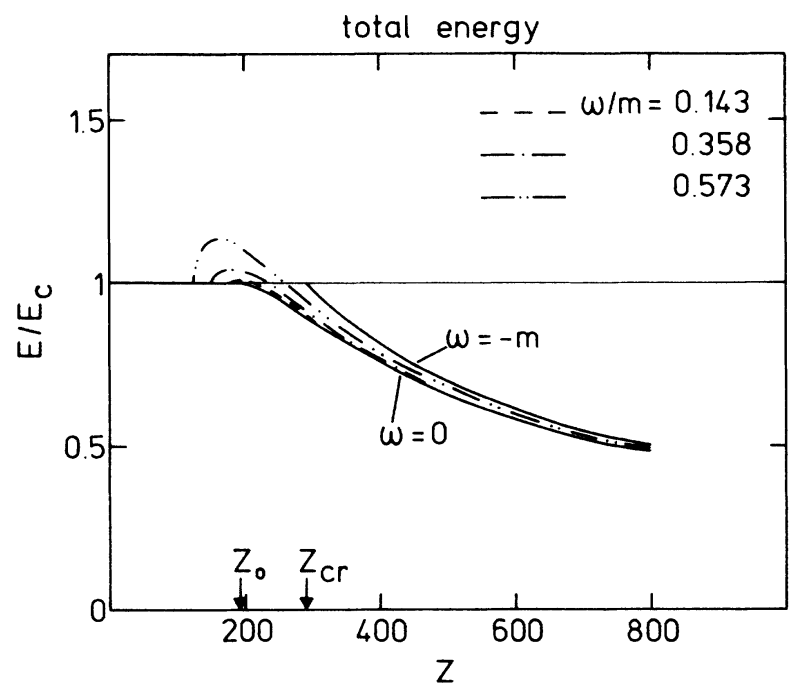

FIG. 2. Values of the total energy $E$ (compared to the Coulomb energy $E_{C}$ ) as a function of the external charge $Z$ for different values of $\omega / \mathrm{m}$. For the sake of clarity, we do not show curves for $0>\omega / m>-1$. All of them start to depart from unity at $Z_{0}<Z<Z_{\mathrm{cr}}$, decrease monotonically, and are comprised between the $\omega=0$ and $\omega=-m$ curves.

The quantities $f_{1}, g_{1}, C$, and $\alpha Q$ are to be determined. Equation (6) also requires

$$
\left.f^{\prime}\right|_{r=r_{0}+\epsilon}-\left.f^{\prime}\right|_{r=r_{0}-\epsilon}=-\frac{\alpha \boldsymbol{Z}}{r_{0}} .
$$

There are only two dimensionless parameters within the model: namely, $x \equiv m r_{0}$ and $\omega / m$. The value of the total charge $Q$ of the external source as a function of $Z$ is plotted in Fig. 1, for a typical $x$ value. One can see that partially screened solutions $(Q<Z)$ exist for any value of

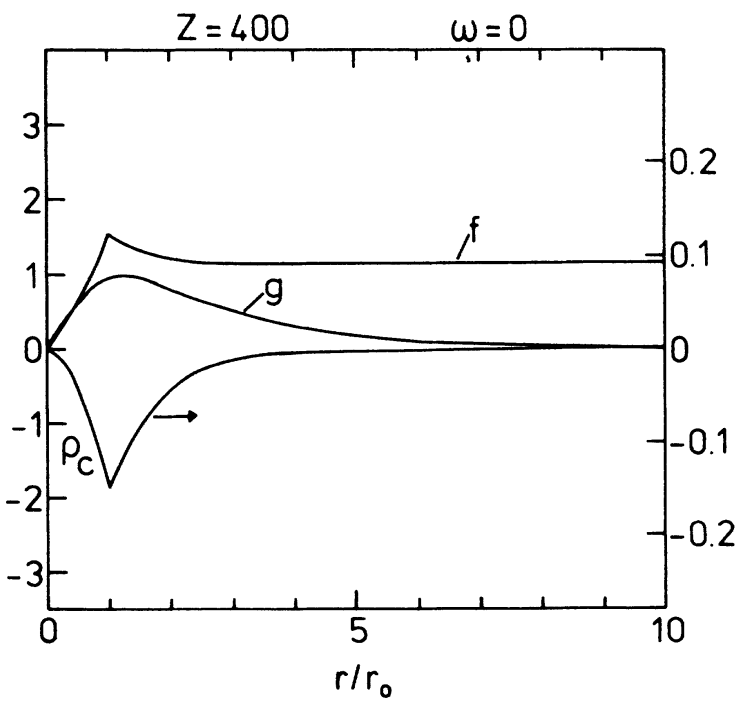

FIG. 3. Shapes of the fields $f$ and $g$ in configuration space for $Z=400, \omega=0$, and $x=0.567$. The curve labeled $\rho_{c}$ gives the charge density of the condensate for this particular case (scale on the right-hand side). 
$Z$. For any value of $\omega$, the screened solution exists for $Z>Z_{\omega}^{0}$, where $Z_{\omega}^{0}$ is the value at which the lowest eigenvalue of the linearized Klein-Gordon equation (12) is equal to $\omega$. However, as shown in Fig. 2, only for $Z>Z_{0}$ [the value at which Eq. (12) has $\omega=0$ solutions] do these solutions have a lower energy than the pure Coulomb ( $g=0)$ solutions. Note that for $Z>Z_{\mathrm{cr}}$, solutions with $\omega=0$ have lower energy and large screening than $\omega / m=-1$ solutions. Figure 3 shows how $f$ and $g$ vary with $r$ for $\omega=0$ and $Z=400>Z_{\mathrm{cr}}$. Also shown is the variation with $r$ of the charge density of the condensate $\rho_{c}$. An expression for $\rho_{c}$ may be derived by integrating Eq. (6) from zero to infinity, after multiplication by $r$. Using (15), one gets

$$
Q=Z+4 \pi \int_{0}^{\infty} r^{2} \rho_{c}(r) d r
$$

with

$$
\rho_{c}=-\frac{1}{4 \pi} \frac{f g^{2}}{r} .
$$

As stated above, the fact that the pure Coulomb solution no longer is the minimum-energy solution for $Z>Z_{0}$ does not imply that it becomes unstable. Actually, our model allows for an exact construction of a classical analogue of the quantum-field-theoretic charged vacuum discussed in Ref. 5. Although it has been recently argued $^{3}$ that such a charged vacuum should not exist in nature, we did not find any support for this thesis in our classical model.

The work of $M$. B. was supported by the National Fund for Scientific Research (Belgium).
${ }^{1}$ M. Bawin and J. Cugnon, Phys. Rev. D 28, 2091 (1983).

2J. E. Mandula, Invited talk of the American Physical Society National Meeting, Chicago, 1977, MIT report (unpublished).

${ }^{3}$ Y. Hirata and H. Minakata, Phys. Rev. D 34, 2493 (1986).

${ }^{4}$ W. Greiner, B. Müller, and J. Rafelski, Quantum Electro- dynamics of Strong Fields (Springer, New York, 1985).

5J. Rafelski, L. P. Fulcher, and A. Klein, Phys. Rep. 38C, 227 (1978).

6J. E. Mandula, Phys. Lett. 68B, 495 (1977). 